\title{
License Plate Detection using OCR with KNN
}

\author{
D. Hareesha, V. Susmitha Rani, S.Gopi Chand
}

\begin{abstract}
This paper presents associate in nursing economical methodology for the detection of vehicle plate by accurately localizing number plates from pictures. A straight forward downscaling of image methodology is projected initial to boost the speed of vehicle plate localization while not reducing the detection performance compared thereupon victimization the first image. Moreover, a completely unique Line Density Filter (LDF) methodology is projected to identify desired regions and eventually reducing the realm to be analysed for vehicle plate detection. Also, a vehicle plate classifier supported SVMs victimization color prominence options employed to spot the vehicle plate from the image. For performance check, a dataset having thirty pictures taken from completely different scenes under different conditions is additionally used. The data set demonstrates that the projected methodology performs well in terms of accuracy and run-time potency.
\end{abstract}

\section{INTRODUCTION}

License Plate Detection (LPD) using OCR with K-Nearest Neighbor (KNN) plays a vital role in several real-life applications, like an automotive vehicle toll assortment, enforcement in traffic, car parking zone access management, police investigation in highways and traffic observance in roads. LPD detects a vehicles registration number from a picture or pictures taken. It's consummated by the mix of a great deal of techniques, like object detection, image processing, pattern recognition and text to speech conversion. Number plate detection is additionally referred to as automatic vehicle identification, and Optical Character Recognition (OCR). License Plate Character Detection is one among the vital technique that introduces indian vehicle registration number plate recognition. Character Recognition stage of the Indian LPR consists a sequence of code characters that needs to be tested with the hold on info, if such a sequence of code characters can be detected, so it could be a valid plate variety [1]. Number plate character recognition strategies square measure generally classified into guide Matching, Neural Networks, and Fuzzy so on. This category of algorithms describes a group of LPCR methods that work effectively for a particular set of image conditions appropriate for Indian License Plates.

Revised Manuscript Received on April 25, 2020.

* Correspondence Author

D. Hareesha*, Assistant Professor, Department of ECE, Prasad V. Potluri Siddhartha Institute of Technology, Kanuru, Vijayawada, India.

V. Susmitha Rani, Department of ECE, Prasad V. Potluri Siddhartha Institute of Technology, Kanuru, Vijayawada, India.

Simhadri Gopi Chand, Department of ECE, Prasad V. Potluri Siddhartha Institute of Technology, Kanuru, Vijayawada, India.

(c) The Authors. Published by Blue Eyes Intelligence Engineering and Sciences Publication (BEIESP). This is an open access article under the CC BY-NC-ND license (http://creativecommons.org/licenses/by-nc-nd/4.0/)
The performance of the projected categories of algorithms has been tested on several live field pictures and also the analysis given by square measure supports the experimental results. This algorithms square measure shows superior results over existing algorithms for Indian License Plates.

\section{WORKING}

Number of object areas within the image are found by using the gray level transitions in it. The windows with a capable range of object areas are shown with left, right, top and bottom line. By using the geometrical details of the detected window like height, breadth and ratio, objects regions are differentiated. Then, range plate is localized and extracted. Finally, options-based matching is utilized for character recognition. The registration code of vehicle will exist any place within the image. The registration code is differentiated by its options, and so the system processes solely the pixels having these options. The options square measure derived from the registration code format and also the characters constituting it. Registration code color is one in every of the options since countries, states or provinces have sure colors for his or her license plates. The oblong form of the registration code boundary is one in every of the feature accustomed. The process is based on extraction of plate region, segmentation of plate characters, recognition of characters and compare the number with database and display the vehicle authorized and status details and give the output in the form of speech. This section presents a method to LPD, which includes preprocessing of image, the extraction of candidate[2] and conversion to speech from text. As shown in Fig 1, the downscaling is done on original color image and then converted into a gray image by the preprocessing procedure. Then, extraction of candidate region via edge detection followed by adaptive thresholding is done. For removal of noise, an LDF is used and segmentation of the image is done. Then, the plate area is cropped and matched with the templates stored in the PC using the K-Nearest Neighbor algorithm [3]. This algorithm is specifically used to reduce the cons of OCR. After matching the characters in the cropped image, the text format of the license number is displayed on the output window. This text is further converted into speech format using the Text To Speech (TTS) methodology.

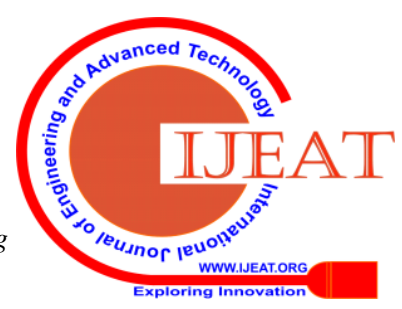




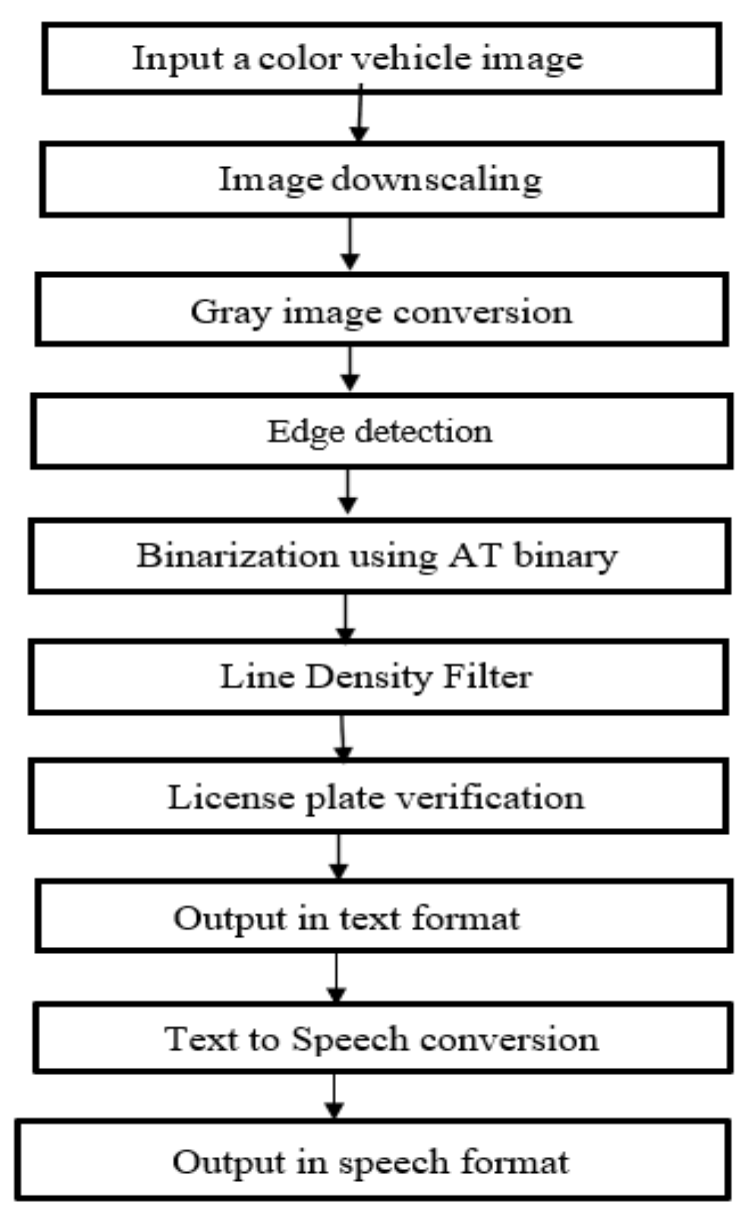

Figure 1: Block Diagram

\section{RESULTS}

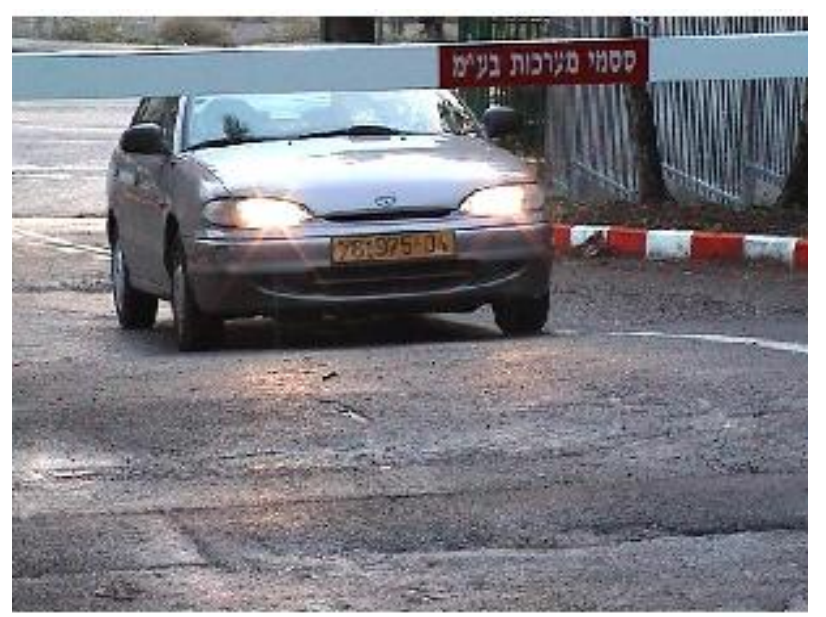

Figure 2: Captured image

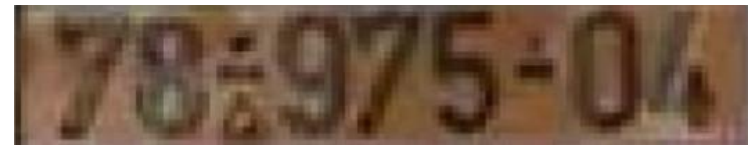

Figure 3: Cropped plate

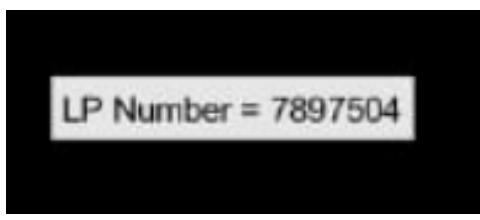

Figure 4: Output text

\section{FURURE SCOPE}

The advances in the technology took the LPD systems which can be used on any PC with software and no additionally expensive hardware is needed. By using this approach with real time images, it can be used in law enforcement vehicles. Now, certain limits on parameters like script on the vehicle license plate, skew in the image can be fixed by enhancing the method.

\section{CONCLUSION}

In this paper, the License Plate Detection using OCR with $\mathrm{KNN}$ is presented. This system was designed by using image processing for recognizing the vehicle from the image. This system works better for different types of conditions. This system was implemented and run on MATLAB and performance is tested using real time captured images. This analysis explains the probability of detection and recognition of the vehicle number plate.

\section{REFERENCES}

1. Aswathy Mani; Nisy John Panicker: An Efficient Method for License Plate Detection and Recognition using OCR (2019). International Journal of Scientific Research in Computer Science Engineering and Information Technology. 540-547. 10.32628/CSEIT1953155.

2. 2.Yule Yuan; Wenbin Zou; Yong Zhao; Xinan Wang; Xuefeng Hu; Nikos Komodakis: A Robust and Efficient Approach to License Plate Detection , IEEE Transactions on Image Processing; Volume-26 Issue-3,

March,2017;Page(s): 1102 -1114;10.1109/TIP.2016.2631901

3. K. Akila, B. Sabitha, R. Jayamurugan, M. Teveshvar, N. Vignesh: Automated License Plate Recognition System using Computer Vision. International Journal of Engineering and Advanced Technology (IJEAT) ISSN: 2249 - 8958, Volume-8 Issue-6, August, 2019.

\section{AUTHORS PROFILE}

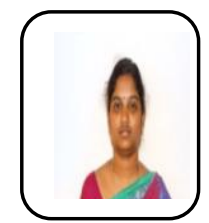

D. Hareesha received UG degree from PGP College of Engg. \& Tech., Namakkal, Tamilnadu. She received PG degree in the stream of Communications and Signa Processing from VRSEC, Vijayawada, Andhra Pradesh. Currently, she is working as Assistant Professor in the Department of ECE, Prasad V. Potluri Siddhartha Institute of Technology, Kanuru, Vijayawada. Her areas of interest include signal processing and image processing. She is a member of IETE \& ISTE.

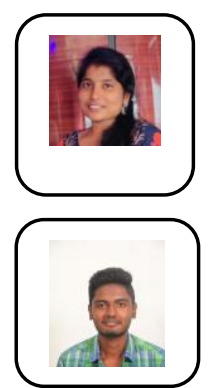

V. Susmitha Rani is pursuing Bachelor of Technology in the Department of ECE, Prasad V.Potluri Siddhartha Institute of Technology, Kanuru, Vijayawada. She is a member of the IETE Student Forum.

Simhadri Gopi Chand is pursuing Bachelor of Technology in the department of ECE, Prasad V.Potluri Siddhartha Institute of Technology, Kanuru, Vijayawada. He is member of IETE Student Forum.

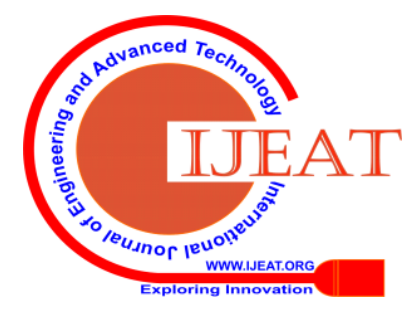

